\title{
Urinary tract infection caused by Chromobacterium violaceum
}

This article was published in the following Dove Press journal:

International Journal of General Medicine

10 September 2015

Number of times this article has been viewed

\section{Narayan Dutt Pant \\ Manisha Sharma \\ Department of Microbiology, Grande International Hospital, Dhapasi, Kathmandu, Nepal}

Correspondence: Narayan Dutt Pant Department of Microbiology, Grande International Hospital, Dhapasi, Kathmandu, Nepal Tel +9779749008524 Email ndpant1987@gmail.com

\begin{abstract}
Chromobacterium violaceum, a proteobacterium, is a facultative anaerobe, which is generally present as the normal flora of water and soil in tropical and subtropical regions. The infection due to Chromobacterium violaceum is rare but mostly fatal. It is responsible for causing fatal cases of septicemia, visceral abscesses, skin and soft tissue infections, meningitis, diarrhea, and rarely urinary tract infection. The bacteria has high propensity to spread causing sepsis. Delayed proper treatment due to limited awareness related to the $C$. violaceum infection is responsible for the high mortality rate. Here, we describe a rare case of urinary tract infection by $C$. violaceum in a chronic kidney disease patient, which was managed with timely proper antimicrobial therapy as per the culture sensitivity report.
\end{abstract}

Keywords: Chromobacterium violaceum, proteobacterium, septicemia, visceral abscesses, nephrectomy

\section{Introduction}

Chromobacterium violaceum is a Gram-negative, facultative anaerobic, oxidase positive, motile bacillus. ${ }^{1}$ It is ubiquitously present in the water and soil of tropical and subtropical regions. C. violaceum was first identified in 1881, and its pathogenic ability was first described by Woolley in 1905 in a fatal infection of a buffalo. ${ }^{2}$ It is well-known for producing violacein, a violet colored pigment with antioxidant property. ${ }^{3}$ C. violaceum rarely infects humans causing skin lesions, sepsis, and liver abscesses that may be fatal. ${ }^{4}$ Other manifestations are soft tissue infections, urinary tract infections, and diarrhea. The first human case of $C$. violaceum infection was reported from Malaysia in 1927 . Worldwide only 150 cases have been reported, mainly from tropics, including eight cases from neighboring country India. ${ }^{5}$ Here, we report a case of urinary tract infection caused by $C$. violaceum in a kidney patient of age 70 years. This is the first case of $C$. violaceum infection reported from Nepal.

\section{Case report}

Written informed consent was obtained from the patient for publication of this case report. A 70-year-old male chronic kidney patient attended the outpatient department of a tertiary care hospital in Kathmandu, Nepal, in November, 2014, with chief complaints of burning micturition, high-grade fever $\left(>102^{\circ} \mathrm{F}\right)$ with chills since 10 days, and pain in lower abdomen. The patient had a history of simple nephrectomy (12 years before) and a recent history of recurrent urinary tract infection. Four weeks ago, the patient was treated for urinary tract infection caused by Escherichia coli. A surgery was done approximately 3 weeks ago for urinary tract obstruction followed by urinary catheterization. 
The patient was empirically treated with ciprofloxacin for urinary tract infection after suggesting necessary laboratory investigation. The dose for ciprofloxacin was adjusted according to the age and the renal condition of the patient. Laboratory findings showed raised total leukocyte count $\left(15,330\right.$ cells $\left./ \mathrm{mm}^{3}\right)$ with neutrophilia $(78.5 \%)$, raised creatinine $(2.1 \mathrm{mg} / \mathrm{dL})$, decreased sodium $(132 \mathrm{mmol} / \mathrm{L})$, and decreased hemoglobin $(13 \mathrm{~g} \%)$. There were plenty of pus cells per low power field with bacteriuria in routine examination of urine.

The mid stream urine culture plated on cystine lactose electrolyte deficient agar as shown in Figure 1 below showed significant growth of single type of nonlactose fermenting colonies after overnight aerobic incubation at $37^{\circ} \mathrm{C}$. The bacterial colonies grown were around $2-3 \mathrm{~mm}$ in diameter with distinctly purple nondiffusible pigment, round, convex with entire margin, opaque, moist, easily emulsifiable, smooth in texture, and dull in appearance. From the colonies grown, the Gram's stain and biochemical tests were performed. The bacterium was Gram-negative motile rod, catalase and oxidase producing, nitrate reducing. It did not produce indole and urease but utilized citrate. It was nonlactose fermenting in triple sugar iron agar. It fermented glucose, trehalose, $n$-acetylglucosamine, and gluconate but did not ferment L-arabinose, D-galactose, or D-maltose and decarboxylated arginine. With the help of these biochemical properties, colony characteristics, and pigment production, it was identified as $C$. violaceum.

Antimicrobial susceptibility testing was performed by Kirby Bauer's disk diffusion technique. The organism was

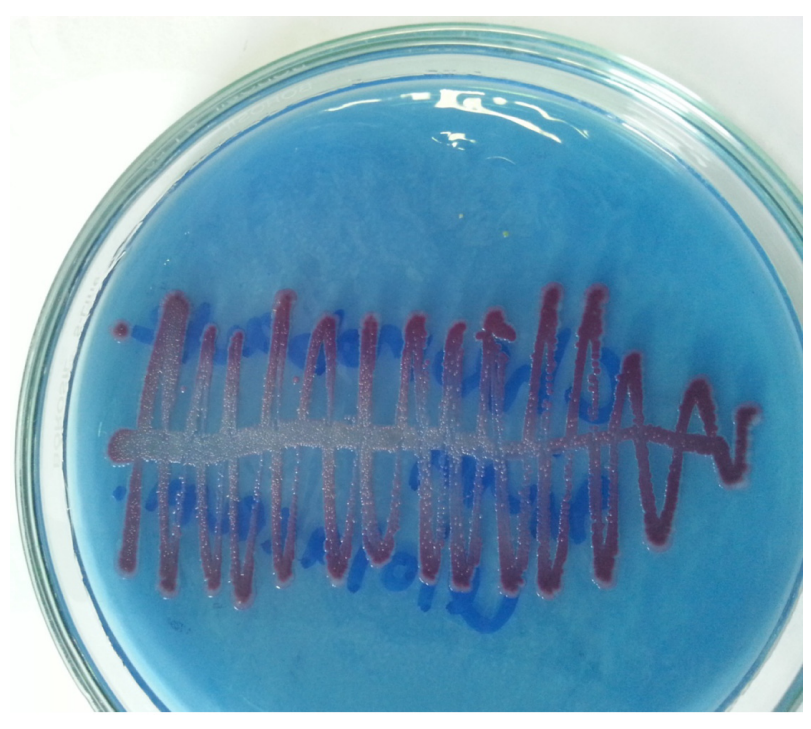

Figure I Growth of Chromobacterium violaceum in CLED agar. Abbreviation: CLED, cystine lactose electrolyte deficient. found to be susceptible toward cotrimoxazole, gentamicin, ciprofloxacin, norfloxacin, imipenem, ceftriaxone and resistant toward amoxicillin and clavulanic acid, and nitrofurantoin. Since the organism was found to be sensitive toward ciprofloxacin, the initial antibiotic was continued for 1 week. Blood culture was done to rule out blood stream infection due to the capacity of the organism for easy dissemination, and the blood culture was negative. After 1 week of the antibiotic administration, the clinical symptoms subsided significantly and the repeated urine culture was negative.

\section{Discussion}

Due to rare infections caused in human by this organism, the awareness of the diseases by $C$. violaceum is limited. ${ }^{6}$ In general, the illness is followed by contaminated inoculation site, which leads to localized disease, regional lymphadenopathy, then hematogenous spread to visceral organs. ${ }^{7}$ It may be responsible for pneumonia, gastrointestinal infection, localized cutaneous lesions, localized or metastatic abscesses, osteomyelitis, meningitis, peritonitis, brain abscess, endocarditis, hemophagocytic syndrome, respiratory distress syndrome, and fulminant sepsis. ${ }^{8}$ Rapid progression to sepsis and multiorgan dysfunction with high mortality rate is a characteristic of $C$. violaceum infection. Urinary tract infection caused by this organism is rarely reported as in 81-year-old male chronic kidney disease patient ${ }^{9}$ and 19 -year healthy male. It is considered as a bacterium of low virulence causing infection in immuno-compromised patients. ${ }^{10}$ In our case also, the patient was immuno-compromised chronic kidney disease patient.

Being mesophilic and ubiquitous in tropical and subtropical areas, it causes infection in summer months in these areas. In addition, this microorganism may not be confined to a narrow geographic range (between latitudes $35^{\circ} \mathrm{N}$ and $35^{\circ} \mathrm{S}$ ) as previously considered because of the effect of global climate change. ${ }^{3,11,12}$ The usual route of the bacteria into the body is through skin trauma or ingestion of contaminated water and seafood. Unusual routes include infection after scuba diving or near drowning, after breast surgery, and appendicectomy. ${ }^{10}$ In our case, the patient had undergone surgery for urinary tract obstruction followed by catheterization. So these may be the causes for the bacterium to get into the urinary tract of the patient. Although antimicrobial susceptibility data on C. violaceum remain very limited because this pathogen is rarely isolated from clinical specimens, results of susceptibility testing vary in different clinical settings. ${ }^{8} \mathrm{C}$. violaceum is usually sensitive to fluoroquinolones, cotrimoxazole, imipenem, gentamicin, ${ }^{13}$ and extremely resistant to penicillins 
and cephalosporins. ${ }^{10}$ In our study also, the organism was found to be sensitive to cotrimoxazole, gentamicin, ciprofloxacin, norfloxacin, imipenem and resistant toward amoxicillin and clavulanic acid.

If the accurate diagnosis is not established promptly and the required treatment is not given in time, the complication may develop and the outcome may be fatal. However, in our case, the patient got well without developing any complications due to timely identification of the causative agent and prompt start of proper antimicrobial therapy as suggested by the urine culture and sensitivity report.

\section{Conclusion}

C. violaceum infection is an emerging infection. If not treated appropriately, it may develop into fatal sepsis. So, the clinician should be aware of this infection and its effective treatment. Urinary tract infection is rarely caused by C. violaceum, but the prognosis may be bad if not managed promptly. So early diagnosis with optimal and adequate antimicrobial therapy for adequate duration is the key to successful treatment.

\section{Disclosure}

The authors report no conflicts of interest in this work.

\section{References}

1. Campbell JI, Lan NP, Qui PT, Dung le T, Farrar JJ, Baker S. A successful antimicrobial regime for Chromobacterium violaceum induced Bacteraemia. BMC Infect Dis. 2013;13:4.

2. Woolley PG. Bacillus violaceous manila, a pathogenic organism. Bull Johns Hopkins Hosp. 1905;16:89-93.
3. Ponte R, Jenkins SG. Fatal Chromobacterium violaceum infections associated with exposure to stagnant waters. Pediatr Infect Dis J. 1992;11(7):583-586.

4. Sneath PH, Whelan JP, Bhagwan Singh R, Edwards D. Fatal infection by Chromobacterium violaceum. Lancet. 1953;265(6780):276-277.

5. Ray P, Sharma J, Marak RS, et al. Chromobacterium violaceum septicaemia from North India. Indian J Med Res. 2004;120(6): 523-526.

6. Chattopadhyay A, Kumar V, Bhat N, Rao P. Chromobacterium violaceum infection: a rare but frequently fatal disease. J Pediatr Surg. 2002;37(1):108-110.

7. Fisher RG, Gruber WC, Boyce TG. Miscellaneous non-enterobacteriaceae fermentative bacilli. In: Feigin RD, Cherry JD, Demmler GJ, Kaplan SL, editors. Textbook of Pediatric Infectious Diseases. 5th ed. Philadelphia: Saunders; 2004:1539-1540.

8. Yang CH, Li YH. Chromobacterium violaceum infection: a clinical review of an important but neglected infection. J Chin Med Assoc. 2011;74(10):435-441.

9. Ma T, Shi W, Cheng J, et al. Chromobacterium violaceum infection in China: three case reports and literature reviews. Afr J Microbiol Res. 2011;5(20):3096-3102.

10. Swain B, Otta S, Sahu KK, Panda K, Rout S. Urinary tract infection by Chromobacterium violaceum. J Clin Diagn Res. 2014;8(8):1-2.

11. Koburger JA, May SO. Isolation of Chromobacterium spp. from foods, soil, and water. Appl Environ Microbiol. 1982;44(6):1463-1465.

12. Byamukama D, Farnleitner AH, Kansiime F, Manafi M, Burtscher M, Mach RL. Contrasting occurrence of Chromobacterium violaceum in tropical drinking water springs of Uganda. J Water Heath. 2005;3(3): 229-238.

13. Aldridge KE, Valainis GT, Sanders CV. Comparison of the in vitro activity of ciprofloxacin and 24 other antimicrobial agents against clinical strains of Chromobacterium violaceum. Diagn Microbiol Infect Dis. 1988;10(1):31-39.
International Journal of General Medicine

\section{Publish your work in this journal}

The International Journal of General Medicine is an international, peer-reviewed open-access journal that focuses on general and internal medicine, pathogenesis, epidemiology, diagnosis, monitoring and treatment protocols. The journal is characterized by the rapid reporting of reviews, original research and clinical studies across all disease areas.

\section{Dovepress}

A key focus is the elucidation of disease processes and management protocols resulting in improved outcomes for the patient. The manuscript management system is completely online and includes a very quick and fair peer-review system. Visit http://www.dovepress.com/ testimonials.php to read real quotes from published authors. 